\title{
Body Weight Reason For Exclusion From
} Statistics

National Cancer Institute

\section{Source}

National Cancer Institute. Body Weight Reason For Exclusion From Statistics. NCI

Thesaurus. Code C119787.

A rationale for excluding a particular data point or subject from the body weight statistical analysis. 